\title{
A new species of Bloxamia from freshwater in the Netherlands
}

\section{Spooren $\mathbf{M}^{1}$}

\author{
${ }^{1}$ Fahrenheitstraat 12, 5621 BR Eindhoven, The Netherlands. E-mail marcos1959@tele2.nl
}

Spooren M 2014 - A new species of Bloxamia from freshwater in the Netherlands. Mycosphere 5(2), 346-349, Doi 10.5943/mycosphere/5/2/8

\begin{abstract}
Collection of a coelomycete belonging to the genus Bloxamia, on submerged stem litter of the Cyperoid plants, Schoenoplectus lacustris, S. tabernaemontani and Eleocharis palustris near Eindhoven, the Netherlands, revealed an undescribed species. The new fungus is described and illustrated herein as Bloxamia hesterae sp. nov. and compared with other species in the genus.
\end{abstract}

Key words - Aquatic fungi - asexual fungi - Europe - helophytes

\section{Introduction}

Freshwater habitats around Eindhoven, the Netherlands, have been scarely surveyed for fungi (Shearer \& Raja 2014) . Massariosphaeria fridae M. Spooren is the only hitherto described taxon from submerged substrates near Eindhoven (Spooren 2007). During studies of freshwater ascomycetes in aquatic habitats near Eindhoven, a species with morphological similarities to the genus Bloxamia was encountered on partly submerged Cyperoid helophytes. The taxon is distinct from previously described species of Bloxamia, and therefore, described and illustrated here as a new species.

\section{Materials and methods}

Fresh, submerged decaying litter was collected in a plastic bag and brought to the laboratory. Crush mounts were made from fresh and air-dried material in $10 \% \mathrm{KOH}$. The slide mount was examined under a Novex microscope (K-series) and a Motic preparation microscope. The photomicrograph was made with a Cmex 1500 scanner. Measurements were made with an image-focus software. The holotype and isotype-materials are deposited in National Herbarium of the Netherlands, Leiden (L) and part material is maintained in the author's personal herbarium.

\section{Taxonomy}

Bloxamia hesterae M Spooren sp. nov.

Figs. 1-5

MycoBank 807930

Holotype - L 0819814

Etymology - hesterae, in memory of Hester (1962-2010), my dear friend who loved sun, sea and flowers.

Saprobic, aero-aquatic, filamentous. Conidiomata 250-500 $\mu \mathrm{m}$ diam., sporodochial, black, superficial, loosely gregarious to scattered, stromatic, cusion-shaped and gelatinous when fresh, and flat, black, corneous and shiny when dry, finally crumbling into a slimy mass. Basal stroma 

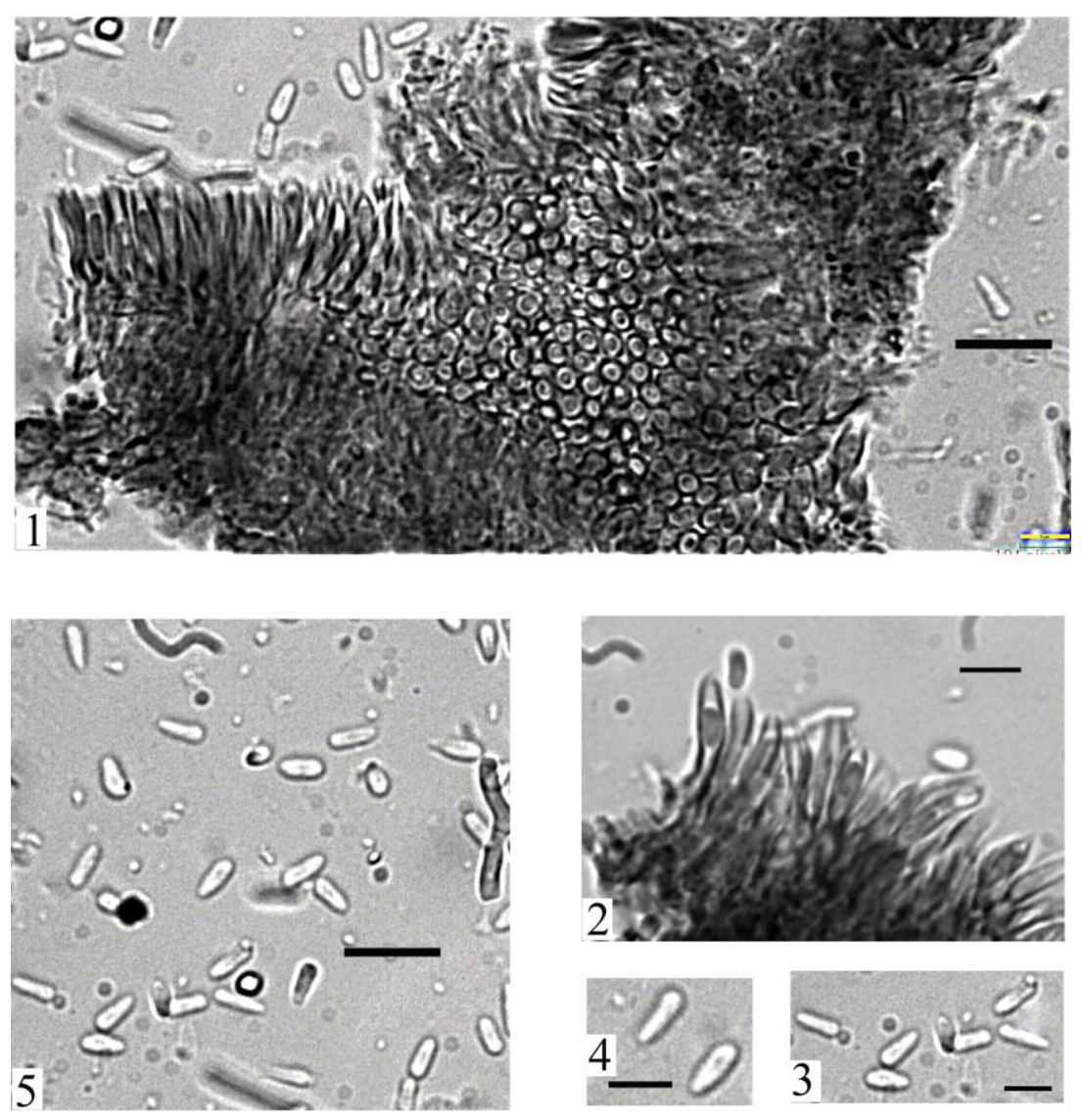

Figs 1-5 - Bloxamia hesterae. 1, Part of the sporodochium with conidiogenous cels from aside and above. 2 , conidiogenous cells with collarette. $3,4,5$ conidia. Scale bars $1=10 \mu \mathrm{m}, 2-5=5 \mu \mathrm{m}$.

barely visible due to the opaque and gelatinous sporodochial mass. Conidiophores macronematous, unbranched, densely and parallely aggregated, terminating in integrated conidiogenous cells.

Conidiogenous cells 14-24 $\mu \mathrm{m}$ long $\times 2-3 \mu \mathrm{m}$ wide, monophialidic, each with $1 \mu \mathrm{m}$ wide constriction at the neck and terminating in a tubular, deep collarette $2-3 \mu \mathrm{m}$ wide $\times 8-1-\mu \mathrm{m}$ long. The collarette closed when young, opens to release ripe conidia at maturity. Conidia 5-6 $\mu \mathrm{m} \times 2-3$ $\mu \mathrm{m}$, endogenous, oblong to clavate, hyaline, aseptate, smooth, slimy. Sexual state unknown.

Specimens examined - Netherlands, Acht, Eindhoven, 23 June 2013, on submerged litter of Schoenoplectus tabernaemontani, M. Spooren (L 0819814, holotype; L 0819815, L 0819816 isotypes); Karperven, Eindhoven, The Netherlands, on pieces of floating stems of Scoenoplectus lacustris, 29 May 2012 (MS 12051); 14 August 2012 (MS 12143); 16 June 2013 (MS 13103); Eindhoven pool near Eindhoven Airport, on submerged stems of Eleocharis palustris, 24 September 2013 (MS 13261); other additional material is maintained in the author's personal herbarium (MS).

\section{Discussion}

The genus Bloxamia Berkeley \& Broome (Berkeley \& Broome 1854), typified by $B$. truncata Berkeley \& Broome, is characterised by its phialophores, densely aggregated in a black sporodochium and arising from a basal thin, prosenchymatous stroma (Ellis 1971, Pirozynski \& Morgan-Jones 1968). The conidiophores are macronematous, erect, cylindrical, septate, sparcely branched, subhyaline, pale brown or brown, forming a palisade over the stroma and terminating in phialides. The conidiogenous cells are phialidic, cylindrical to subcylindrical, with a deep collarette extending from undifferentiated venter. The phialoconidia are endogenously differentiated, short cylindrical to cuboid, oblong with truncate ends, and unicellular, hyaline to subhyaline.(Nag Raj \& Kendrick 1975). 
These latter authors based their description on two, sporodochial type species, B. truncata Berk and Br. (=B. leucopthalma (Lev.) Hohn.) and the synnematal species B. nilagirica (Subram.) Nag Raj \& Kendrick. Another synnematal species, B. foliicola Yun L. Liu \& Z.Y Zhang, was subsequently described from China, as a leaf parasite (Liu \& Zhang 1998).

Three other species are described in Bloxamia: B. bohemica Minter \& Hol.-Jech. (Minter \& Holubová.-Jechova 1981), with cylindrical conidiophores and cylindric conidia, isolated from Pinus needle litter; B. cremea Arambarri, Cabello \& Cazau (Arambari et al. 1992), with white sporodochia and cylindrical conidia, from decaying bark of an unknown plant. The closest species to B. hesterae is however B. sanctae-insulae Coppins \& Minter (Coppins \& Minter 1981), which has a widened colarette in the conidiogenous cell, but differs in that the conidiophores are less deep, inflated and tapering towards the apex and the conidia are globose or with a very small hilum. B. sanctae-insulae has been reported on wood or bark of an unknown tree. Johnston (1988) described a Chalara-like asexual state for Bisporella discedens (Karst.) Carpenter which he assigned to Bloxamia because of the conidiogenous cells develop in sporodochia.

For a comparison see table 1.

Table 1 Comparation of all described Bloxamia species.

\begin{tabular}{|c|c|c|c|}
\hline & Conidiomata & Conidiogenous cells & Conidia \\
\hline B. nilagirica & $\begin{array}{l}\text { Synnematal } \\
\text { brown } \\
1120-1260 \mu \mathrm{m} \text { long } \\
\text { base } 260-380 \mu \mathrm{m} \\
\text { apex } 140-220 \mu \mathrm{m}\end{array}$ & & $\begin{array}{l}\text { Hyaline } \\
\text { rectangular } \\
4-5,5 \times 3-3,5 \mu \mathrm{m} \\
\text { long and slimy chain }\end{array}$ \\
\hline B. foliicola & $\begin{array}{l}\text { Synnematal } \\
\text { brown } \\
670-860 \mu \mathrm{m} \text { long } \\
\text { base } 46-103 \mu \mathrm{m} \\
\text { apex } 64-152 \mu \mathrm{m}\end{array}$ & $\begin{array}{l}\text { Branched } \\
\text { cylindrical } \\
\text { brown } \\
64-95 \times 10-11 \mu \mathrm{m}\end{array}$ & $\begin{array}{l}\text { Hyaline } \\
\text { nearly square } \\
\text { both ends truncate } \\
6-9 \times 5-8 \mu \mathrm{m} \\
\text { dry chain. }\end{array}$ \\
\hline B. cremea & $\begin{array}{l}\text { Sporodochial } \\
\text { white to cream } \\
500-1000 \mu \mathrm{m}\end{array}$ & $\begin{array}{l}\text { Branched } \\
\text { cylindrical } \\
\text { dark brown } \\
24-26 \times 2,5-3 \mu \mathrm{m}\end{array}$ & $\begin{array}{l}\text { Hyaline } \\
\text { cylindrical } \\
3-4 \times 1,5 \mu \mathrm{m} \\
\text { long and slimy chain }\end{array}$ \\
\hline B. truncata & $\begin{array}{l}\text { Sporodochial } \\
\text { black } \\
140-180(-500) \mu \mathrm{m}\end{array}$ & $\begin{array}{l}\text { Simple } \\
\text { cylindrical to subcylindrical } \\
\text { pale brown } \\
15-32 \times 2-3 \mu \mathrm{m}\end{array}$ & $\begin{array}{l}\text { Hyaline-subhyaline } \\
\text { short cylindrical to oblong } \\
\text { rounded apex, truncate base } \\
\text { (or both ends obtuse ) } \\
2-4(-7) \times 1,5-2,5 \mu \mathrm{m} \\
\text { single or easily dispersable } \\
\text { chain }\end{array}$ \\
\hline B. bohemica & $\begin{array}{l}\text { Sporodochial } \\
\text { amber, greenish when wet } \\
2000 \times 1000 \mu \mathrm{m}\end{array}$ & $\begin{array}{l}\text { Simple } \\
\text { lageniform } \\
\text { pale brown } \\
8-11 \times 1,5-2 \mu \mathrm{m} \\
\text { Collarette: cylindrical } \\
8-11 \times 1,5 \mu \mathrm{m}\end{array}$ & $\begin{array}{l}\text { Hyaline } \\
\text { cylindrical } \\
3-5,5 \times 1 \mu \mathrm{m} \\
\text { in chains }\end{array}$ \\
\hline B. sanctae-insulae & $\begin{array}{l}\text { Sporodochial } \\
\text { brown to black } \\
1500 \times 750 \mu \mathrm{m}\end{array}$ & $\begin{array}{l}\text { Simple } \\
\text { lageniform } \\
\text { pale brown } \\
10-14 \times 1,5-2,5 \mu \mathrm{m} \\
\text { Collarette: cylindrical } \\
5-7 \times 1,5-2,5 \mu \mathrm{m}\end{array}$ & $\begin{array}{l}\text { Hyaline } \\
\text { globose or with small hilum } \\
2 \mu \mathrm{m} \text { diam. } \\
\text { in chains }\end{array}$ \\
\hline B. hesterae & $\begin{array}{l}\text { Sporodochial } \\
\text { opaque black } \\
250-500 \mu \mathrm{m} \text { diam. }\end{array}$ & $\begin{array}{l}\text { Simple } \\
\text { lageniform } \\
\text { black } \\
14-24 \times 2-3 \mu \mathrm{m} \\
\text { Collarette: cylindrical } \\
8-10 \times 2-3 \mu \mathrm{m}\end{array}$ & $\begin{array}{l}\text { Hyaline } \\
\text { oblong to clavate } \\
5-6 \times 2-3 \\
\text { single, slimy }\end{array}$ \\
\hline
\end{tabular}




\section{Acknowledgements}

I wish to thank Drs. Vadim Mel'nik, Huzefa Raja and Christian Scheuer for critical reading and commenting the drafts of this article.

\section{References}

Arambarri A, Cabello M, Cazau C. 1992 - A new hyphomycete from santiago river. V. Bloxamia cremea. Mycotaxon 43 327-330.

Berkeley M.J, Broome, CE. 1854 - Notices of British fungi (730-784). Annals and Magazine of Natural History. 13:458-469

Coppins BJ, Minter DW. 1981 - A new hyphomycete from Northhumberland. Notes RBG Edinb.38: 363-365.

Ellis MB. 1971 - Dematiaceous Hyphomycetes. Commonwealth Mycological Institute, Kew, Surrey, England.

Johnston P. 1988 - The Bloxamia anamorph of Bisporella discedens. Mycotaxon 31: 345-350.

Liu Yun-Long, Zhang Zhong-Yi.1998 - A new species of the genus Bloxamia. Mycosystema 17: 710.

Minter DW, Holubová-Jechová V. 1981 - New or interesting Hyphomycetes on decaying pine litter from Czechoslovakia. Folia Geobot.Phytotax., Praha 16: 195-217.

Nag Raj TR, Kendrick B. 1975 - A Monograph of Chalara and Allied Genera.Wilfried Laurier University Press Waterloo, Ontario, Canada.

Pirozynski KA, Morgan-Jones G. 1968.- Notes on microfungi III. Trans. Br. Mycol. Soc. 51: 185206

Shearer CA, Raja HA. Freshwater Ascomycetes and their Anamorphs: http://fungi.life.illinois.edu/. Accessed Jan 5, 2014.

Spooren M. 2007 - Massariosphaeria fridae, a new freshwater ascomycete. Persoonia 19: 261263. 